\title{
Simulated Lunar Testing of Metabolic heat regenerated Temperature Swing Adsorption
}

\author{
Sebastian A. Padilla ${ }^{1}$, Chad E. Bower ${ }^{2}$ and Christie S. Iacomini ${ }^{3}$ \\ Paragon Space Development Corp , Tucson, Arizona 85714 \\ and \\ Heather L. Paul ${ }^{4}$ \\ NASA Johnson Space Center, Houston, Texas, 77058
}

\begin{abstract}
Metabolic heat regenerated Temperature Swing Adsorption (MTSA) technology is being developed for thermal and carbon dioxide $\left(\mathrm{CO}_{2}\right)$ control for a Portable Life Support System (PLSS), as well as water recycling. An Engineering Development Unit (EDU) of the MTSA Subassembly (MTSAS) was designed and assembled for optimized Martian operations, but also meets requirements for lunar operations. For lunar operations the MTSA sorption cycle is driven via a vacuum swing between suit ventilation loop pressure and lunar vacuum. The focus of this effort was testing in a simulated lunar environment. This environment was simulated in Paragon's ECLSS Human-rating Facility (EHF) chamber. The objective of the testing was to evaluate the full cycle performance of the MTSAS EDU, and to assess $\mathrm{CO}_{2}$ loading and pressure drop of the wash coated aluminum reticulated foam sorbent bed. Lunar environment testing proved out the feasibility of pure vacuum swing operation, making MTSA a technology that can be tested and used on the Moon prior to going to Mars. Testing demonstrated better than expected $\mathrm{CO}_{2}$ loading on the sorbent and nearly replicates the equilibrium data from the sorbent manufacturer. This exceeded any of the previous sorbent loading tests performed by Paragon. Subsequently, the increased performance of the sorbent bed design indicates future designs will require less mass and volume than the current EDU rendering MTSA as very competitive for Martian PLSS applications.
\end{abstract}

\section{Nomenclature}

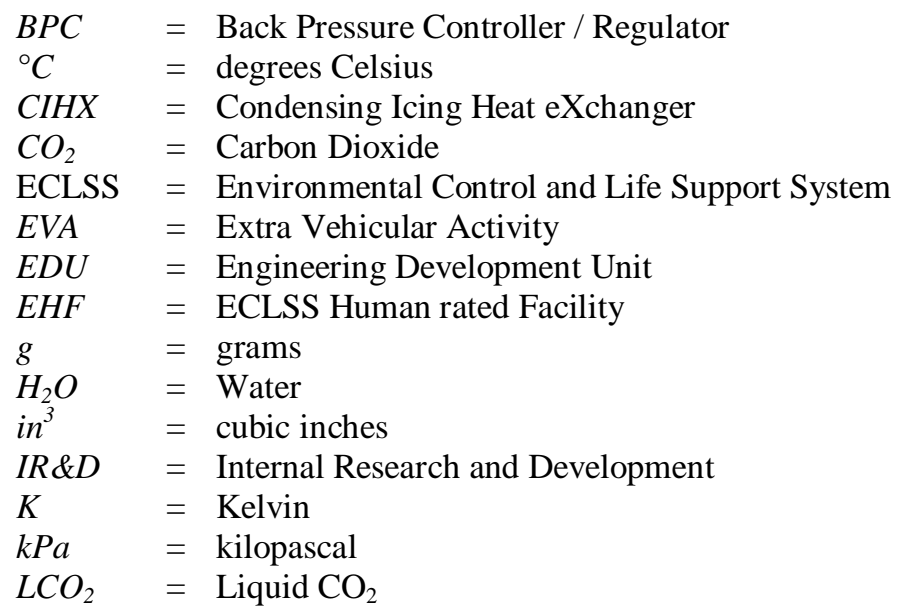

${ }^{1}$ MTSA Project Lead, 3481 E. Michigan Street Tucson, Arizona, 85714, AIAA Senior Member Grade.

${ }^{2}$ Sr. Thermal Analyst, 3481 E. Michigan Street Tucson, Arizona, 85714, AIAA Member Grade.

${ }^{3}$ Principal Investigator, 3481 E. Michigan Street Tucson, Arizona, 85714, AIAA Senior Member Grade.

${ }^{4}$ Space Suit PLSS Project Engineer, Crew and Thermal Systems Division, 2101 NASA Parkway, Mail Code EC8 


$\begin{array}{ll}L C V G & =\text { Liquid Cooling \& Ventilation Garment } \\ M F C & =\text { Mass Flow Controller } \\ m g / s & =\text { milligrams per second } \\ M T S A S & =\text { MTSA Subassembly } \\ N_{2} & =\text { Nitrogen } \\ O_{2} & =\text { Oxygen } \\ P L S S & =\text { Portable Life Support System } \\ P P C O_{2} & =\text { Partial Pressure } \mathrm{CO}_{2} \\ \text { ppm } & =\text { Parts Per Million } \\ S & =\text { Seconds } \\ S L P M & =\text { Standard Liters Per Minute } \\ S H X & =\text { Sublimation Heat eXchanger } \\ W & =\text { Watts }\end{array}$

\section{Introduction}

$\mathrm{M}$ etabolic heat-regenerated Temperature Swing Adsorption (MTSA) is patent-pending (USPTO 61222208) technology, being developed for Portable Life Support Subsystem (PLSS) carbon dioxide $\left(\mathrm{CO}_{2}\right)$ removal and rejection as well as thermal regulation and humidity control. The metabolically-produced $\mathrm{CO}_{2}$ present in the ventilation loop gas of a PLSS is collected using a $\mathrm{CO}_{2}$-selective sorbent via temperature swing adsorption. The temperature swing is achieved through cooling using Martian extracted liquid $\mathrm{CO}_{2}\left(\mathrm{LCO}_{2}\right)$ and warming using heat from ventilation loop gas used by the astronaut. Figure 1 illustrates how an MTSA subsystem would be operated in a PLSS using two sorbent beds. Each bed is cycled between adsorb and desorb mode. The concept and its development history has been described previously in detail, ${ }^{1,2,3,4}$ but is summarized briefly here as well.

A schematic demonstrating how the MTSA can be employed in a PLSS is shown in Figure 1. Ventilation gas returning from the astronaut enters the PLSS. Metabolic heat and humidity are first removed from the ventilation loop (on the left) via the Condensing Icing Heat eXchanger (CIHX) in contact with the cold sorbent bed fully loaded with metabolically-produced $\mathrm{CO}_{2}$. Water condenses out of the ventilation gas and initially freezes. The trapped metabolically-produced $\mathrm{CO}_{2}$ in the sorbent is rejected to ambient as the bed is warmed (straight red arrow pointing down on left). Meanwhile, as the bed continues to warm (to $\sim 280 \mathrm{~K}$ ), the ice thaws inside the CIHX and condensate is saved.

The ventilation gas exiting the CIHX is now cooler and drier. A recuperative membrane and desiccant will be required to remove any remaining moisture (water can limit the sorbent's $\mathrm{CO}_{2}$ loading capacity). Passing through the second bed, metabolically-produced $\mathrm{CO}_{2}$ is removed from the ventilation gas by the sorbent. To increase the capacity of the sorbent in the second bed, the sorbent is cooled with coolant via the Sublimation Heat eXchanger (SHX) (blue lines pointing up on right). Coolant gas exhaust is further used with the liquid cooling ventilation garment (LCVG) for thermal control before being rejected to the mostly- $\mathrm{CO}_{2}$ Martian atmosphere.

Regenerated, pure oxygen ventilation gas exits the sorbent bed. A recuperative heat exchanger is used to warm the ventilation gas prior to return to the astronaut. Lastly, the dry line is humidified with the membrane recuperative humidifier.

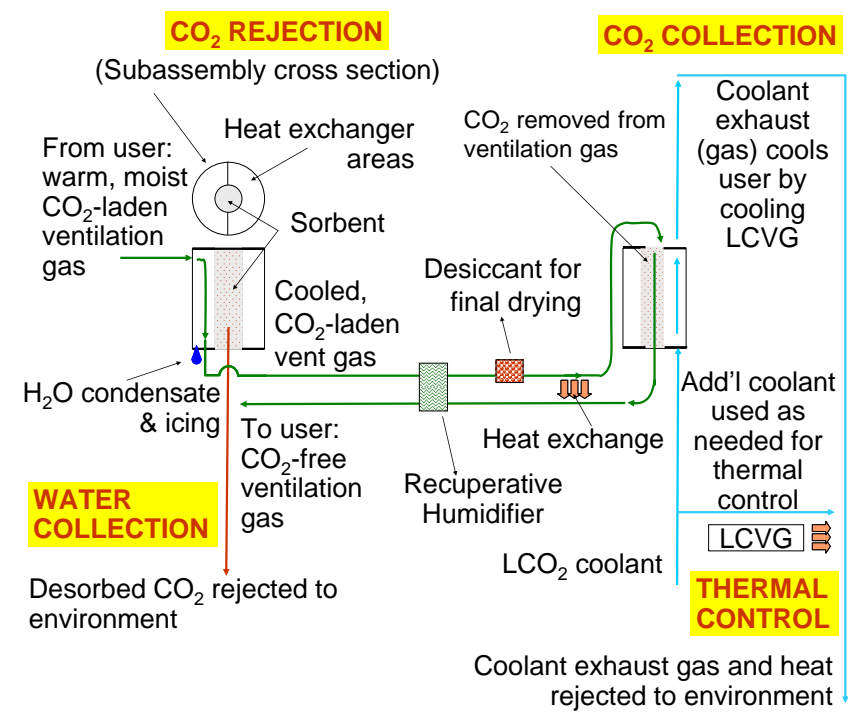

Figure 1. Two bed MTSA system operation.

Continuous removal of metabolically-produced $\mathrm{CO}_{2}$ is achieved using two beds that cycle between desorb mode $\left(\mathrm{CO}_{2}\right.$ rejection) and adsorb mode $\left(\mathrm{CO}_{2}\right.$ collection). Each bed will perform the same loading and unloading cycles as shown in Figure 2. Figure 2 (left) demonstrates how each bed works in adsorb and desorb modes to ensure continuous $\mathrm{CO}_{2}$ removal. Figure 2 (right) demonstrates how the $\mathrm{CO}_{2}$ loading changes with temperature and pressure within a given bed for a Martian application. 

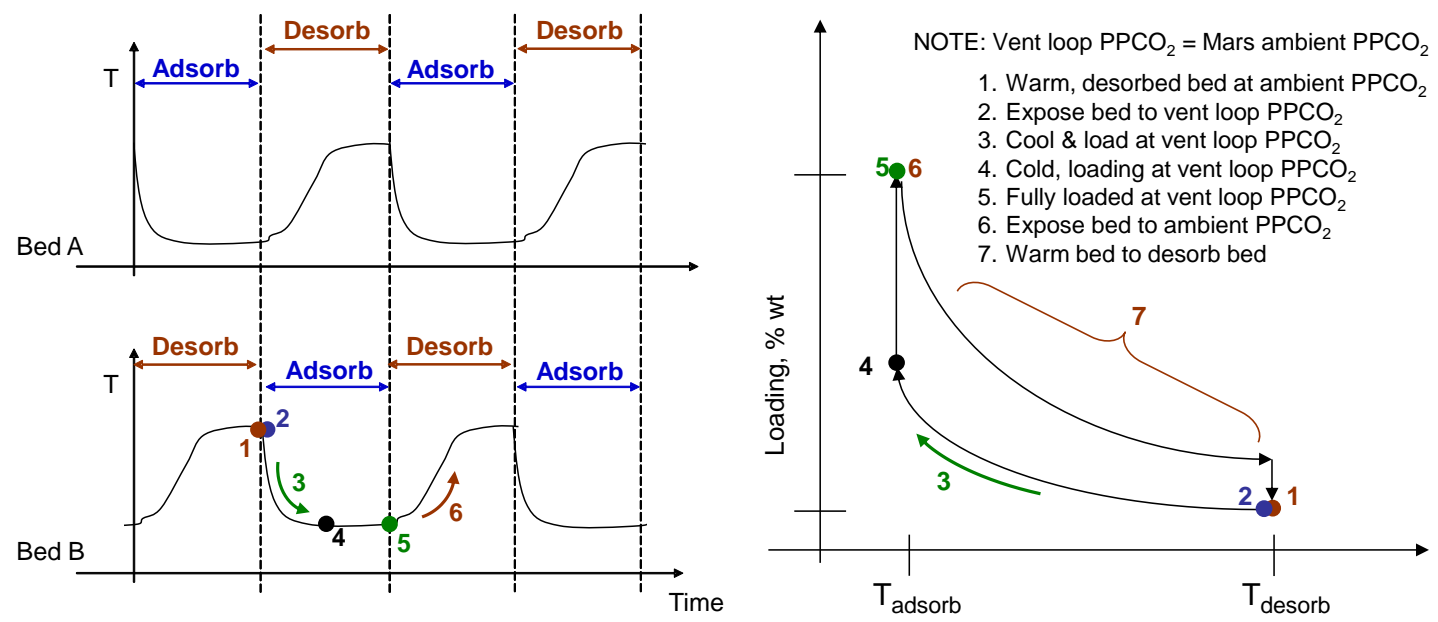

Figure 2. (Left) Loading cycles (temperature vs time); (right) \% Loading changes vs temperature.

MTSA was originally designed for Mars using $\mathrm{LCO}_{2}$ coolant derived from the Martian atmosphere. Production of $\mathrm{LCO}_{2}$ on Mars from the readily available $\mathrm{CO}_{2}$ atmosphere can be achieved for relatively low power using the cold Martian nights to facilitate the process. This is a tremendous mass savings and reduction in mission risk because missions do not have to rely on the coolant being launched from Earth. Additionally, as $\mathrm{LCO}_{2}$ is not cryogenic, reserves of $\mathrm{LCO}_{2}$ can be stored on the surface of Mars with no risk of boil-off. To extend an EVA or obtain emergency cooling, it is only necessary to switch out or refill the $\mathrm{LCO}_{2}$ tank. Finally, as the cooling capacity of the $\mathrm{LCO}_{2}$ is consumed, its exhaust can be safely expelled to the Martian atmosphere where it does not contaminate the surrounding environment (the Martian atmosphere is $95 \% \mathrm{CO}_{2}$ ). Thus, a Martian PLSS that uses MTSA will not interfere with scientific investigations by contaminating samples with water vapor as its coolant (the Martianderived $\mathrm{CO}_{2}$ ) is sublimated for heat rejection.

MTSA is also a means for risk mitigation because it does not have PLSS technologies in common with the current spacesuit PLSS baseline. This means that heat rejection and ventilation loop $\mathrm{CO}_{2} \&$ humidity control are all handled completely differently than the current baseline. As MTSA technology addresses well the challenges posed by missions performed in the unique environment of Mars, with very limited accessibility from Earth, pursuing MTSA is sound justification for mitigating PLSS development risk.

In addition, the design can theoretically be used on the Moon with no modifications to the sorbent bed. The lunar vacuum can be used to regenerate the sorbent bed alone via a vacuum swing. This reduces the amount of coolant required and uses the moon as a test bed for furthering Mars technology development, but will require an as of yet unidentified means of drying the ventilation loop gas. Previous testing had always been performed in a Martian

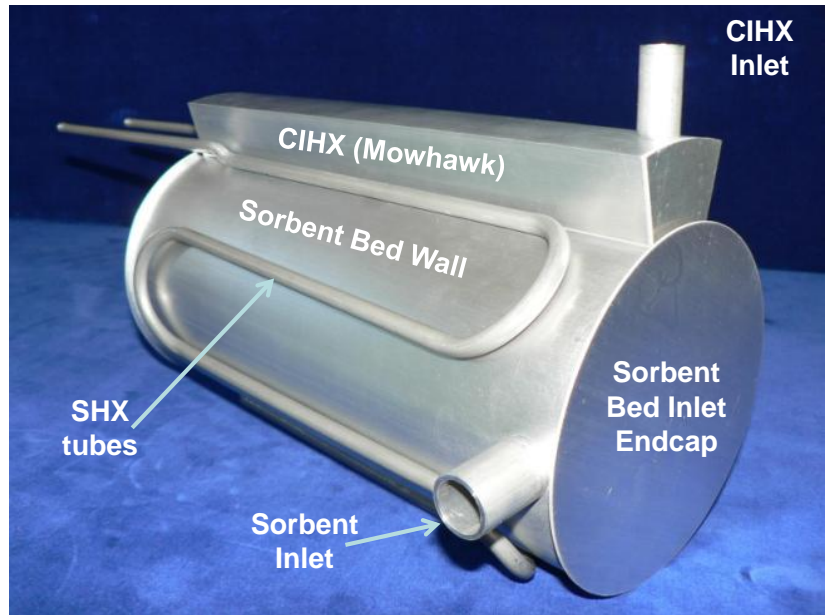

Figure 3. Engineering Development Unit of the safety. environment, ${ }^{5}$ the current effort aims to demonstrate performance in a simulated Lunar environment.

\section{A. Problem Statement and Goals}

The overall objective of this testing effort was to evaluate the performance of a full scale MTSAS EDU in a simulated lunar vacuum environment across the full range of metabolic loading. This information could then be used to compare to system modeling efforts of the wash coated foam ${ }^{6}$ and provide evidence of the efficacy of using such an MTSAS design in a lunar environment. The objective is to assess $\mathrm{CO}_{2}$ loading and pressure drop for the wash coated aluminum foam sorbent bed within a Mars-capable MTSAS EDU design using "nominal" MTSA operating conditions with a $\mathrm{N}_{2} / \mathrm{CO}_{2}$ mixture representative of ventilation loop gas. $\mathrm{N}_{2}$ is substituted for $\mathrm{O}_{2}$ to reduce complexity and increase MTSAS 


\section{Lunar Testing}

The manufactured EDU is shown in Figure 3 (previous page), where both the CIHX and Sorbent bed exhaust ports are on the back side of the unit and not visible in the figure. The design and manufacture is summarized in Ref. 7. The sorbent bed forms the base, cylindrical structure around which everything else is attached. For Martian operations, the CIHX, through which the moist, $\mathrm{CO}_{2}$ laden ventilation loop gas flows, is comprised of a "Mohawk" shaped structure attached to the top of the structure. Lastly, the SHX, through which the $\mathrm{LCO}_{2}$ coolant flows, snakes along the outside cylindrical wall of the sorbent bed. For lunar operation, the CIHX and SHX are not required.

\section{A. Test Description}

The test bed for this effort is largely based on the one used and adapted for much of the previous MTSA testing $^{5,8,9}$ as well as the EHF test bed created under a Paragon IR\&D effort to upgrade the existing test bed. One driving factor for modifications of the previous bed for this work revolves around the need to simulate lunar vacuum conditions. Paragon's Environmental Control and Life Support System (ECLSS) Human-rating Facility (EHF) is a $180 \mathrm{ft}^{3}$ vacuum chamber which pulls a vacuum on the exterior of the EDU, which, provides a sink for the desorbed $\mathrm{CO}_{2}$. A schematic of the test bed is shown in Figure 4.

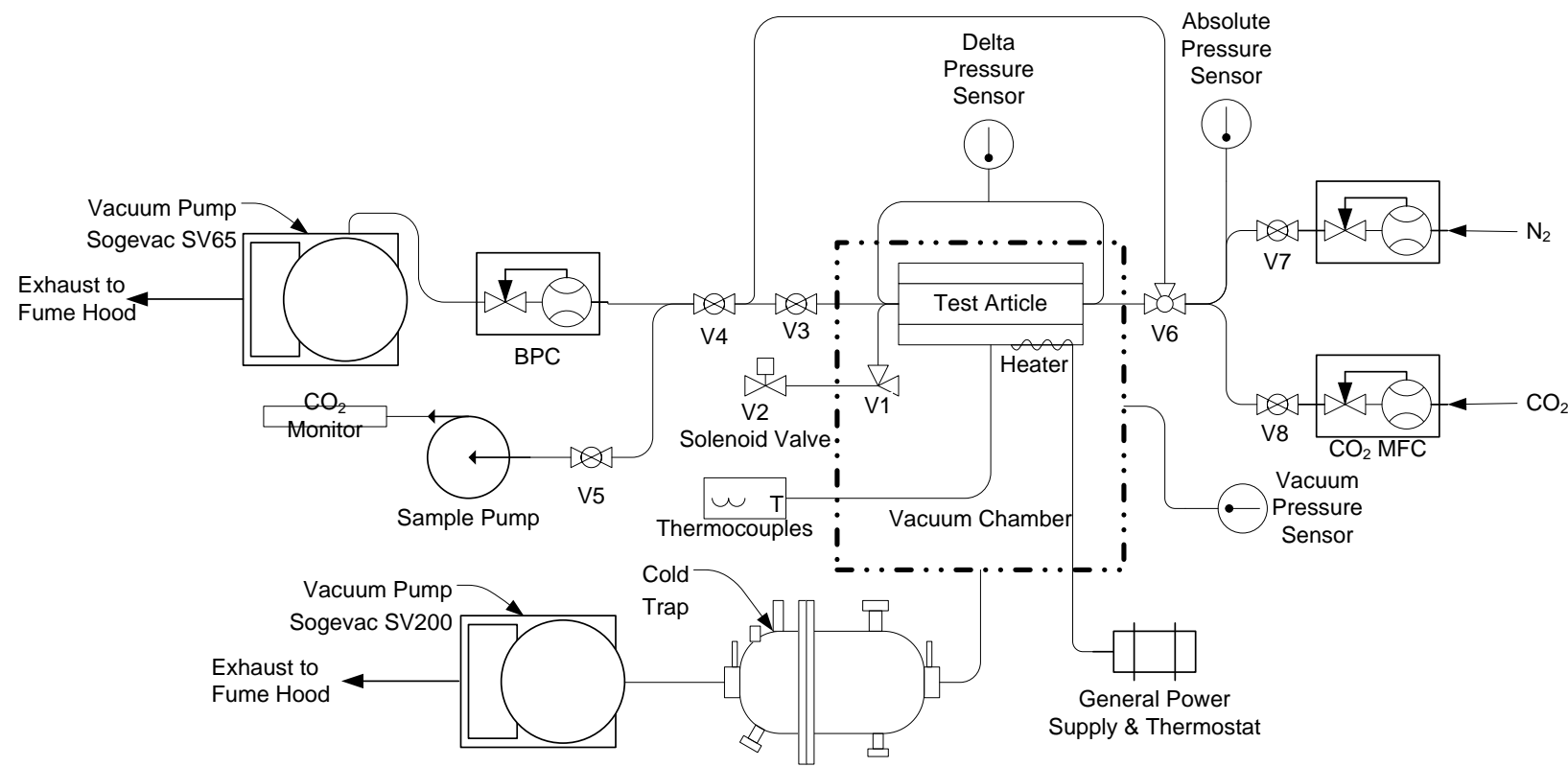

Figure 4. EDU Lunar Environment Test Schematic using the EHF Chamber

The test bed is comprised of the cold trap and pump (SV200), which are attached in series to the EHF chamber with pressure sensors and support equipment for both the test article and the EHF (sealing and connections hardware). A vacuum valve (V1) is placed inside the chamber with pneumatic lines plumbed to it for cycling the supply line to the chamber environment. The overall purpose of the test bed is to pump down the EHF, while cycling a controlled $\mathrm{N}_{2}$ and $\mathrm{CO}_{2}$ flow to a test article or exposing the test article to the internal vacuum. The cold trap and vacuum pump maintain the chamber pressure and a backpressure regulator and additional vacuum pump maintain vent loop pressure for the $\mathrm{N}_{2}$ and $\mathrm{CO}_{2}$ flow.

A prebalanced (through the use of the bypass loop from $\mathrm{V} 6$ to $\mathrm{V} 4$ ) mix of $\mathrm{N} 2$ and $\mathrm{CO}_{2}$ gas is routed to the test article (the MTSA EDU) during the adsorb portion of the cycle. A combination of the SV65 vacuum pump and the Alicat Back Pressure Regulator (BPC) maintain the pressure at 4.3 psia in the test article (as measured by the upstream Absolute Pressure Sensor). The nominal adsorption cycle is run until $\mathrm{CO}_{2}$ breakthrough measures at the maximum 20,000 ppm on the $\mathrm{CO}_{2}$ monitor (measured at $1 \mathrm{~atm}$ of pressure). This is short of the $33,000 \mathrm{ppm}(1 \mathrm{kPa})$ limit levied as a requirement but sufficient for prediction of the breakthrough timing. During the low metabolic profile $100 \mathrm{~W}$ case, the adsorption cycle is run until 8,000 $\mathrm{ppm}(0.6 \mathrm{kPa})$ is reached, which is the sorbent bed inlet $\mathrm{CO}_{2}$ concentration. During desorption the test article is isolated from the $\mathrm{N} 2$ and $\mathrm{CO}_{2}$ flow and exposed to the vacuum within the EHF via the pneumatic vacuum valve (V1). 
Prior to ventilation loop initiation, the EHF must be pumped down to simulate the external lunar environment. Once the internal pressure of the EHF is stable, the ventilation loop flow for adsorption may be initiated. In order to establish the flow properly, a bypass loop is included in the test bed. The vacuum valve within the chamber is closed for the adsorb cycle. Once ready, the flow is directed through the EDU where $\mathrm{CO}_{2}$ is adsorbed for a period of time. Breakthrough is monitored by the $\mathrm{CO}_{2}$ sensor and the flow maintained through the EDU until a predetermined concentration is reached. Once that concentration is met, the flow is switched to bypass and the vacuum valve opened to expose the sorbent bed to the simulated lunar environment. The bed desorbs for a period of time and then the valve is closed; flow is directed back through the EDU to restart the cycle.

\section{B. Test Matrix}

The test matrix is shown in Table 1. For each test case, the test article was run through the adsorb / desorb cycle until the time for the adsorption cycle is within, at most, $10 \%$ of the previous run. The end of the adsorption cycle is

\begin{tabular}{|l|l|l|l|l|l|}
\hline $\begin{array}{l}\text { Test } \\
\text { Case }\end{array}$ & $\begin{array}{c}\text { Metabolic } \\
\text { Rate }\end{array}$ & $\begin{array}{l}\mathbf{N}_{2} \text { Flow } \\
\text { (SLPM) }\end{array}$ & $\begin{array}{l}\mathbf{C O}_{2} \text { Flow } \\
\text { (SLPM) }\end{array}$ & $\begin{array}{l}\mathbf{C O}_{2} \text { concentration } \\
\text { ends Adsorb } \text { 1/2-Cycle }\end{array}$
\end{tabular}
defined as the time coincident with the $\mathrm{CO}_{2}$ sensor reaching the concentration level referenced in Table 1.

The purpose of test case 1 is to define the baseline performance of the system at the nominal metabolic and flow rates. Test case 2 is at the same metabolic rate, but desorbs for twice the adsorb time to determine if the bed is able to reach full desorption at the nominal cycle time. Test case 3 is at the low metabolic rate and will help with characterization of a Thermal Desktop model $^{6}$ as well as show performance of the system at low metabolic rates. Test case 4 is at the high metabolic rate and will help with characterization of the Thermal Desktop model as well as show performance of the system at peak metabolic rates.

\section{Test Bed Design and EDU Integration}

Thermocouples were installed on the MTSAS EDU in 12 locations (see Figure 5).

- 1 Thermocouple (TC) on sorbent bed inlet tube stub: TC01

- $1 \mathrm{TC}$ on sorbent bed outlet tube stub: TC02

- $1 \mathrm{TC}$ on the sorbent bed inlet end-cap in the center: TC03

- 3 TCs on CIHX top, one each on inlet side, center and outlet side. Approximately in center of each third: TC04, TC05, and TC06.

- 5 TCs opposite to CIHX, one each on inlet side, center and outlet side. Approximately in center of each third: TC07, TC08, TC09, TC10, and TC11.

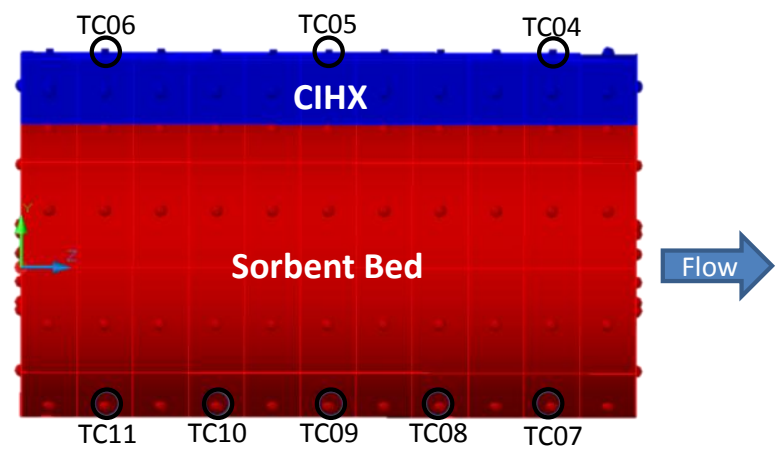

Figure 5. Thermocouple Placement (nodes inside black circles)

- $1 \mathrm{TC}$ on exhaust side of sorbent bed at the interface of the end-cap and sorbent structure tube (for monitoring of S-Bond braze during bake-out): TC12.

\footnotetext{
${ }^{5}$ Maximum allowable $\mathrm{CO}_{2}$ concentration is $33,000 \mathrm{ppm}(1 \mathrm{kPa})$. The sensor max reading is $20,000 \mathrm{ppm}(0.6 \mathrm{kPa})$.

6 Time to complete adsorb cycle is defined as the $1 / 2$-cycle time.
} 


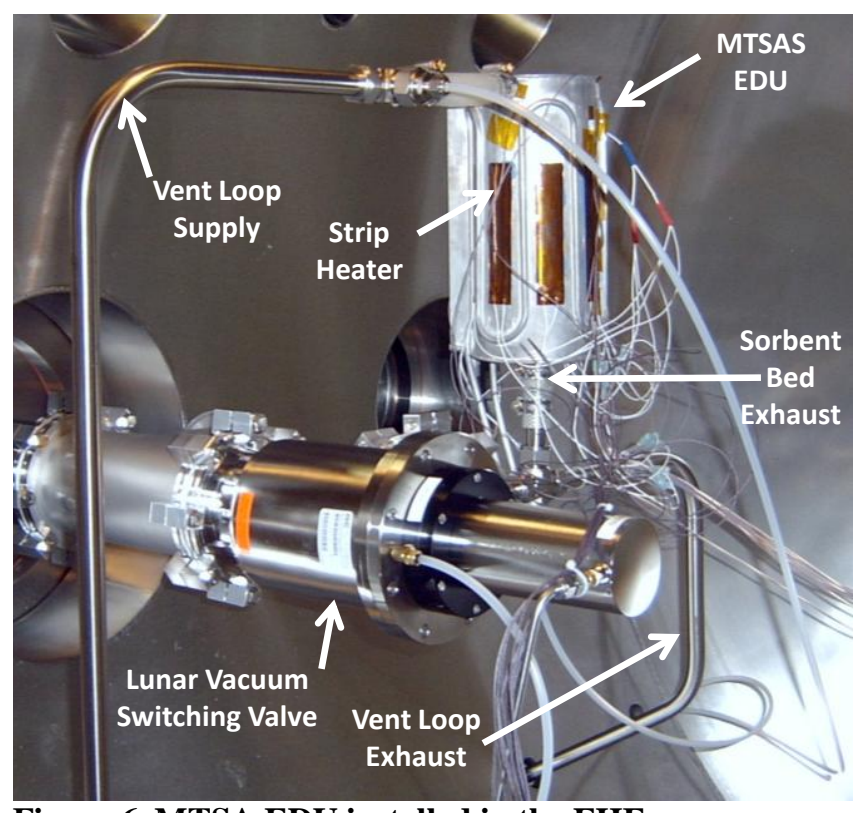

Figure 6. MTSA EDU installed in the EHF

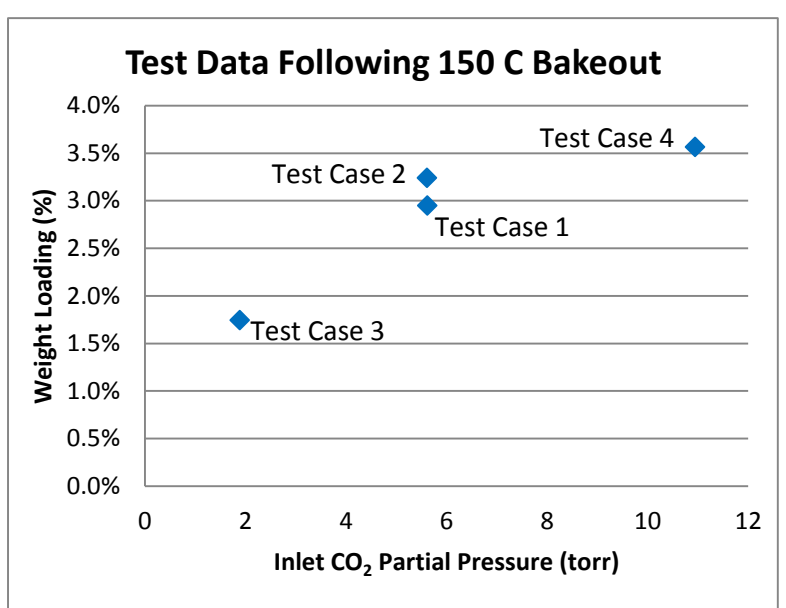

Figure 7. Average, Delta Sorption Loading (uncertainty less than $3 \%$ of value).

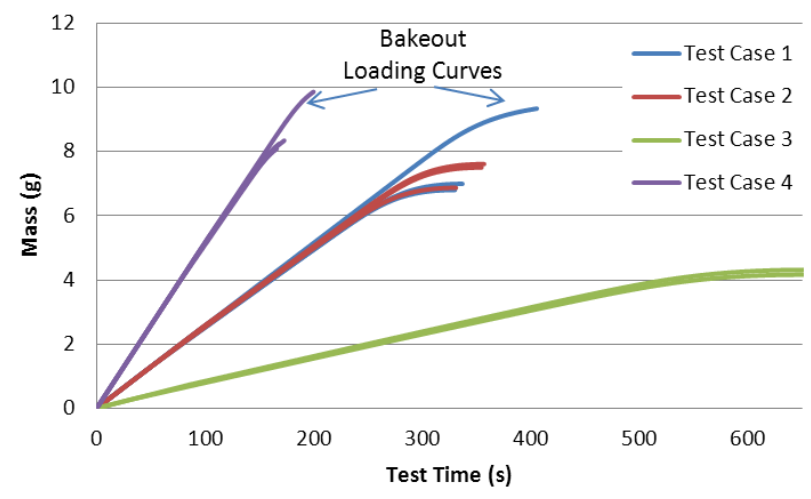

Figure 8. $\mathrm{CO}_{2}$ Adsorption Cycle Mass Loading (Sorbent Prepared with $150^{\circ} \mathrm{C}$ Bake-out)
The MTSA EDU is shown installed within the EHF chamber in Figure 6. Strip heaters were attached to accommodate bakeout and reconditioning of the sorbent bed.

\section{Testing}

Following a bake-out at $150^{\circ} \mathrm{C}$ for 8 hours at 95 $\mathrm{kPa}(13.8 \mathrm{psia})$ and 5 SLPM dry Nitrogen purge flow to condition the sorbent, the test cases shown in Table 1 were run in succession and results are displayed in Figure 7. The data shown represents the cyclic loading capability achieved for test case conditions from Table 1 so that the values should be representative of performance during an EVA. The nominal case with $25.9 \mathrm{mg} / \mathrm{s}$ inlet flow of $\mathrm{CO}_{2}$ (at 6 torr $\mathrm{PPCO}_{2}$ ) resulted in $2.9 \%$ loading which exceeds the cyclic loading value of $2 \%$ that was anticipated for the lunar testing. This is comparable to what is expected to be the maximum possible cyclic loading potential of $3.4 \%$. Note that the higher 6 torr data point at $3.2 \%$ corresponds to the double length desorption time data (test case 2). This data suggests that, for this configuration, adsorption potential is desorption limited at the time scales of these runs ( 350 vs. 750 seconds for desorption, for Test Case 1 and 2 respectively), and indicates the need for further study of the desorption characteristics.

Test Case 3 and Test Case 4 show the dependence of $\mathrm{CO}_{2}$ weight loading on inlet partial pressure of $\mathrm{CO}_{2}$. As one would expect, Test Case 3 with an inlet $\mathrm{CO}_{2}$ partial pressure of approximately 2 torr achieves a weight loading below that of Test Cases 1 and 2, while Test Case 4, with an inlet $\mathrm{CO}_{2}$ partial pressure of approximately 11 torr exceeds the first two test cases.

The calculated mass loading trends for the data set is given in Figure 8. Test case 1 and 2 data have the same loading rate, given the same loading conditions. However, test case 2 accumulates additional $\mathrm{CO}_{2}$ since the desorb time is twice that of test case 1 . The longer desorb time allows for more loading capacity. The first run of test case 1 and 4 were conducted following a bake-out. As before, this data demonstrates that full desorption is not achieved as following cycles have less capacity to adsorb $\mathrm{CO}_{2}$. The effect of the additional desorption time is clear in test case 2 as the test article can adsorb $\mathrm{CO}_{2}$ for a longer time, resulting in higher totals. The slopes of the loading curves in Figure 8 suggest that the sorbent exposed to lower $\mathrm{CO}_{2}$ partial pressure (Test Case 3) is closer to the loading limits for a given ventilation loop gas partial pressure of $\mathrm{CO}_{2}$ than sorbents exposed to higher $\mathrm{CO}_{2}$ flow rates (Test Case 4, for example). As the loading slope approaches zero, the sorbent $\mathrm{CO}_{2}$ accumulation is approaching zero.

The energy of adsorption and desorption is clearly seen by the temperature response of the EDU during 
adsorption / desorption cycling (see Figure 9). The adsorption occurs as a wave, starting at the inlet side of the sorbent bed. Thermocouple TC11 is the first to warm with subsequent thermocouples responding regularly as the adsorption front moves through the bed; TC10 warms next followed by TC09, then TC08 and lastly TC07 warming at the exhaust side of the sorbent bed. This trend is expected from a bed that efficiently loads from bed inlet to bed outlet. On the other hand desorption cooling happens throughout the bed at the same time regardless of thermocouple position; this makes sense as the entire bed rapidly depressurizes when it is exposed to the simulated

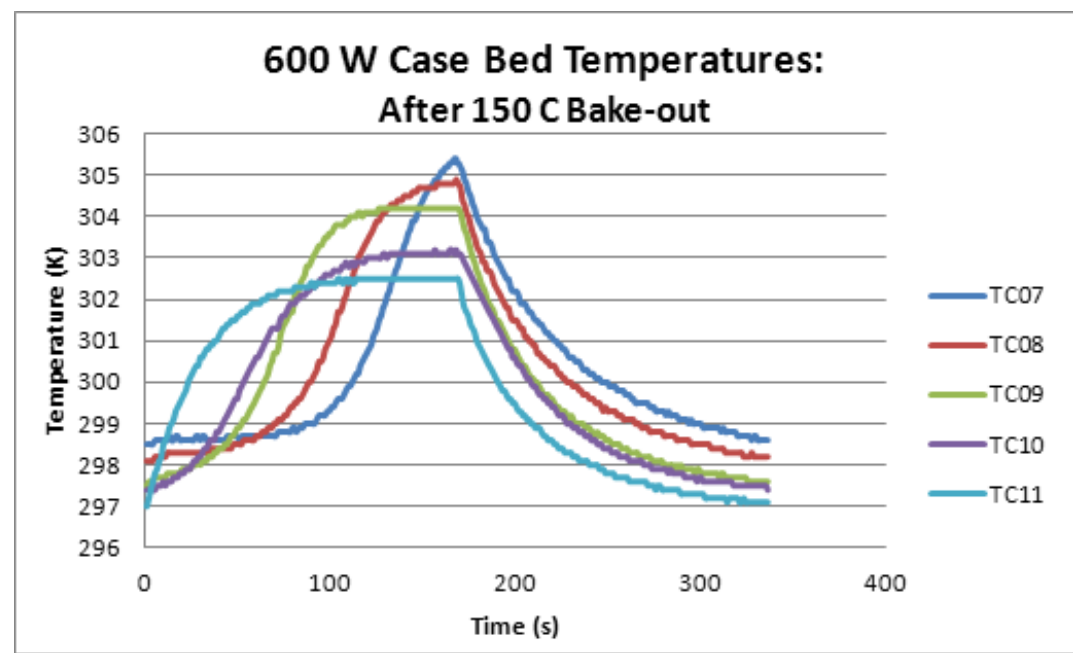

Figure 9. Sorbent Bed Cycle Temperatures lunar vacuum.

Breakthrough of the sorbent bed happens suddenly towards the end of the adsorption cycle. This is clearly demonstrated for test case 1 and 4 in Figure 10. While these curves are for the test cases run immediately after bake-out the curve is representative of each of the curves for test case 1 and 4.

\section{E. Results \& Data Analysis}

While the performance data gathered under the lunar operations testing is not analogous to those in Martian testing, the general performance can be assessed. Figure 11 shows a plot containing data from UOP (the sorbent manufacturer)

containing absolute equilibrium weight loading (rather than the delta weight loading attained in a vacuum swing as shown in Figure 7). The plot also contains data collected in Lunar EDU testing of low, nominal and high metabolic rates. The plotted pressure corresponds to the inlet $\mathrm{CO}_{2}$ partial pressure where higher inlet pressure corresponds to increased metabolic rate since the inlet total mass flow rate is constant. The measured total sorbent load correlates well with the given manufacturer data and even slightly exceeds expectations. This total weight loading data is created by collecting data directly following a full bake out. Since vacuum swing alone is insufficient to remove all of the $\mathrm{CO}_{2}$, the cycle capacity is somewhat lower than these values.

The difference between the adsorbed $\mathrm{CO}_{2}$ following a full bake out and that adsorbed in following cycles is the loading of $\mathrm{CO}_{2}$ that remains in the bed at the end of a desorption cycle, labeled in Figure 11 as the minimum desorption levels. These data were collected only for the nominal and high metabolic rates. These data further make clear that the amount of desorption achieved is a function of the desorption

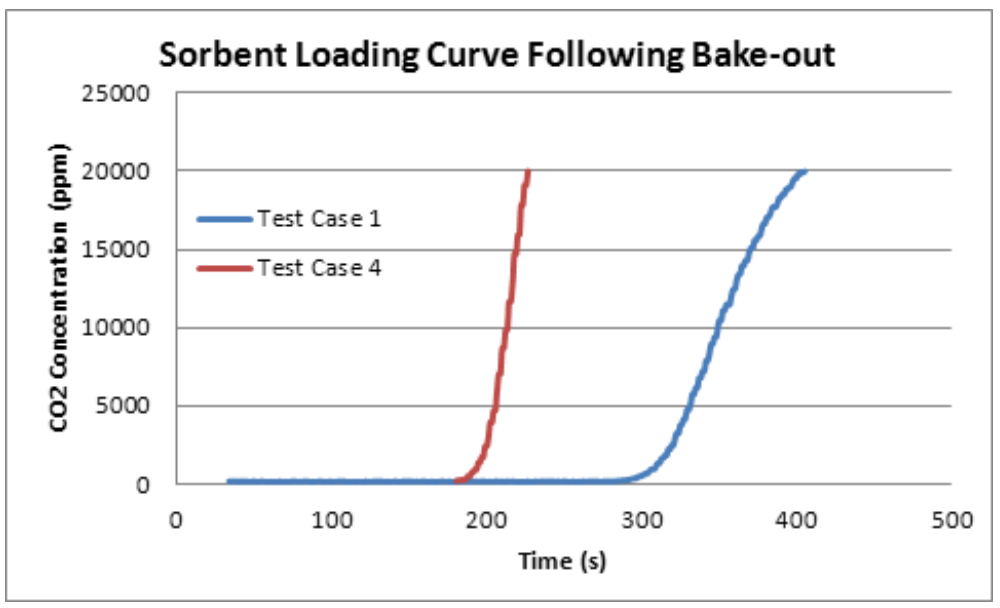

Figure 10. $\mathrm{CO}_{2}$ Breakthrough duration. Since the nominal metabolic $\mathrm{CO}_{2}$

flow rate was tested at both $1 \mathrm{x}$ and $2 \mathrm{x}$ the adsorption time, there are minimum desorption levels plotted for both cases. Since no bake-out was performed between $1 \mathrm{x}$ and $2 \mathrm{x}$ tests, the longer desorption time minimum is calculated by subtracting the difference of the cycle loading percentages. This is thought to be valid since it is expected that the same total loading is achieved, and the only difference in capacity in the two tests is the level of desorption. While no bake out was performed for the low metabolic rate, the desorption time for the case is about the same as that for the $2 \mathrm{x}$ nominal metabolic case. This desorption level is applied to that data. 


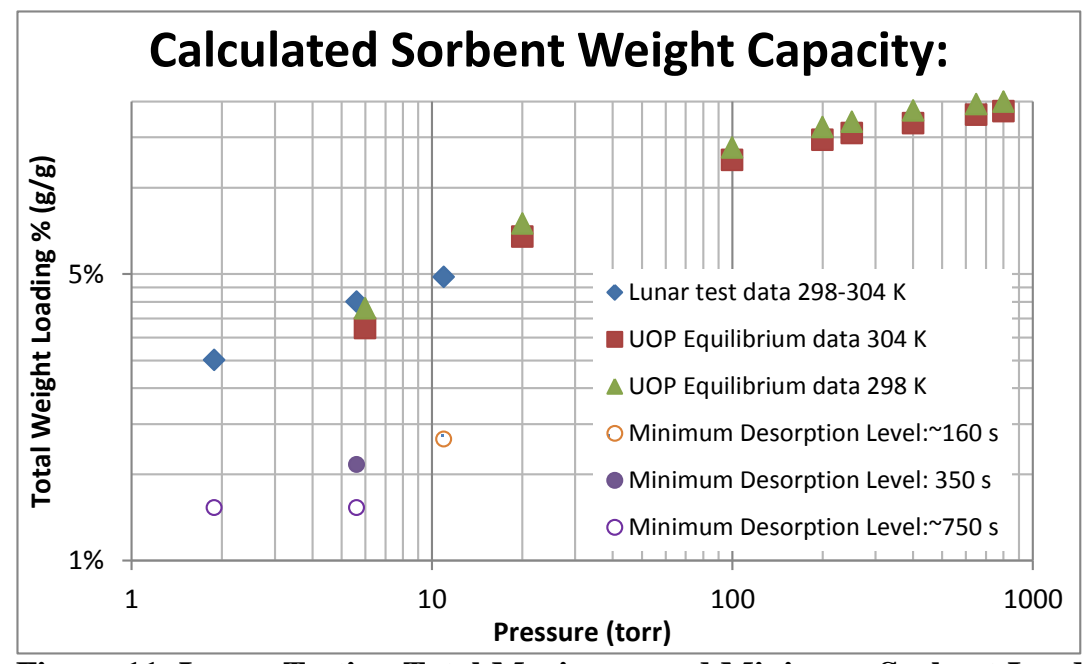

Figure 11. Lunar Testing Total Maximum and Minimum Sorbent Loads and UOP Equilibrium Data (uncertainty less than $3 \%$ of value).
Absolute loading and minimum desorption level data are important in developing accurate models. It is important to note that while the data in Figure 11 are plotted against the manufacturer's equilibrium data, they are not equilibrium data themselves. If the entire bed were exposed to the inlet $\mathrm{CO}_{2}$ concentration for extended periods, the loading may reach a level that exceeds the measured values. This effect can be seen in the data collected in Figure 11. The low metabolic case data far exceeds an extrapolation of the UOP data, the nominal case exceeds by a lesser amount, and the high metabolic rate data falls short of the UOP data.

This trend is expected to a degree since the longer cycle times of low metabolic rates allow more of the bed to approach equilibrium loading. The maximum metabolic rate has less than $1 / 4$ of this time to do so. It should be also noted that as the equilibrium data provided by UOP is for $1 / 8$ inch pellets, the test data may be indicating that the wash coat approach allows for more efficient use of the sorbent mass but this requires more testing to verify.

These data are critical in developing useful models of the sorbent bed. Correlation of the developed models involves developing a prediction for the equilibrium values that allows simulation of each of these results. Once correlated, these estimated equilibrium values can be used to create performance predictions for other geometries, flow rates, or total pressures, allowing for MTSA optimization. This is equally true for the desorption data. It is expected that desorption flow rates follow a regular exponential form of decay. Collected test data for a given design geometry with different desorption times can be leveraged to characterize the desorption process, again allowing the data to be extended to system or specific subassembly design variations.

The gathered lunar test data is helpful in understanding the expected sorbent loading in the Martian testing. An example of the results of the previous wash coated articles tested in Martian conditions at a range of adsorption temperatures are shown in Figure 12. ${ }^{5}$ Total adsorption is shown for a $210 \mathrm{~K}$ data point, with other temperatures assumed to have the same desorption minimum. While this assumption may not be fully correct, it is instructive in comparing data. In terms of total loading potential, the tests achieved only about half of the total expected loading potential at each temperature (the test article data resides approximately

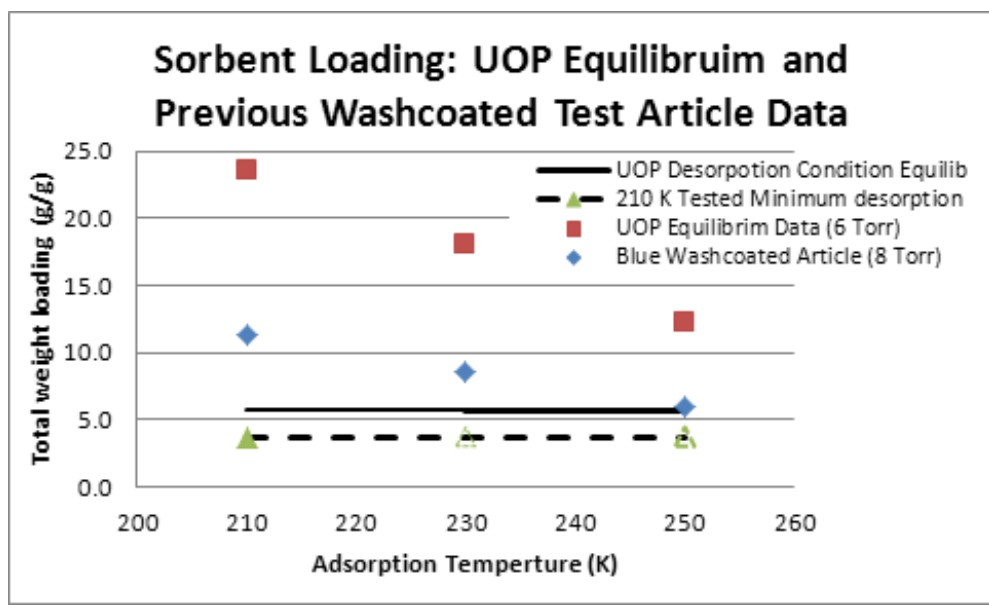

Figure 12. Martian Test Results and UOP Equilibrium Data at 210 $\mathrm{K}, 230 \mathrm{~K}$, and $250 \mathrm{~K}$.

halfway in between the " $210 \mathrm{~K}$ Tested Minimum desorption" line and the "UOP Equilibrium Data" line). Consideration of the tested minimum data in relation to the total captured (the measured cyclic change in loading percentage) falls much faster than the absolute load. This again shows the impact of cycle time in the ability to capture $\mathrm{CO}_{2}$. Extrapolation of this line suggests that the bed would lose the ability to capture $\mathrm{CO}_{2}$ at about $260 \mathrm{~K}$, or a temperature swing of about $20 \mathrm{~K}$ due to ever shortening cycle times at higher temperatures. The inability of the sorbent to efficiently capture the $\mathrm{CO}_{2}$ at shorter cycles in part explains the need for the development and application of sorbent models in MTSA sizing analyses. For point of reference, the $210 \mathrm{~K}$ test point from the previous tests 
required a 50 second run. This is only $1 / 3$ of the cycle time of the $\sim 160$ seconds required for the high metabolic rate lunar test which indicated time related inefficiencies.

This is not to say that short cycle times are fully responsible for adsorption inefficiencies in the previous testing. It is noted that significantly more bridging between pores occurred in the previous test articles than in the EDU which had much improved coating characteristics at an even high density of wash coat. ${ }^{7}$ Fully blocked pores are functionally inaccessible to $\mathrm{CO}_{2}$ over both the adsorption and desorption intervals. Additionally there is always the chance of fouling of the sorbent, which could reduce performance. Any of these three factors (cycle time, bridging, and fouling) would go toward explaining the inability of the test articles to achieve a tested minimum desorption that is higher than the provided equilibrium as is expected.

The good performance of the EDU at the time intervals seen in lunar testing, between 160 and 800 seconds, bode well for the operational feasibility of full cycle operation under Martian operation. While the 160 second lunar test shows some adsorption time related inefficiency and the previous articles indicate this becoming more severe at even shorter cycles, the expected cycle time for nominal metabolic rates is expected to be on the order of 900 seconds or more. At these cycle times, little cycle time duration effect is expected. While it is difficult to extrapolate

\begin{tabular}{|l|c|c|c|c|c|c|c|c|}
\hline & $\begin{array}{c}\text { Sorbent } \\
\text { weight } \\
\text { loading } \\
\text { performance }\end{array}$ & $\begin{array}{c}\text { Sorbent } \\
\text { Loading }\end{array}$ & $\begin{array}{c}\text { Half } \\
\text { cycle } \\
\text { time }\end{array}$ & $\begin{array}{c}\text { Bed } \\
\text { Diameter }\end{array}$ & $\begin{array}{c}\text { Bed } \\
\text { Length }\end{array}$ & $\begin{array}{c}\text { MTSAS } \\
\text { Mass }\end{array}$ & $\begin{array}{c}\text { Desiccant } \\
\text { Mass (+ } \\
\text { container) }\end{array}$ & $\begin{array}{c}\text { Total } \\
\text { mass }\end{array}$ \\
\hline EDU as designed & 11.0 & 1.34 & 12.0 & 4.5 & 9.0 & 1.1 & 0.4 & 1.5 \\
\hline $\begin{array}{l}\text { Coating and } \\
\text { loading optimized } \\
\text { EDU }\end{array}$ & 17.8 & 1.65 & 5.0 & 2.7 & 5.4 & 0.4 & 0.3 & 0.7 \\
\hline
\end{tabular}

Table 2. EDU Design Optimization

lunar test findings to a Martian case due to very different operating conditions, the lunar test may suggest that the combination of improved manufacturing and cycle times may allow nearly the full equilibrium potential of the sorbent to be realized. This is to say that rather than the $9 \%$ delta required, a value as high as $17 \%$ could be achieved. Performance at this level would have a profound impact on the system sizing. Small increases in performance could result in significant reductions in system volume and mass, as well as expendables mass and while maintaining a large performance margin. This can only be confirmed by further testing.

To demonstrate the strong impact of weight loading performance on system parameters the analysis tool developed to optimize EDU design ${ }^{7}$ was exercised with the increased sorbent loading ( $1.65 \mathrm{~g} / \mathrm{in}^{3}$ of sorbent bed $\left.{ }^{7}\right)$ and the full $17.75 \%$ potential suggested by the UOP data. Results are shown in Table 2. This optimized bed shows that there is potential to make an MTSAS that is half the mass and $60 \%$ of the diameter and length than the EDU built for this effort.

\section{Conclusions}

MTSA operation was demonstrated by testing an EDU in a simulated Lunar environment. Several conclusions can be drawn from the effort completed. Most notably, $\mathrm{CO}_{2}$ loading on the sorbent was better than expected and nearly replicates the equilibrium data (see Figure 11) of the sorbent. This had not been achieved in any of the previous MTSA sorbent design loading tests performed by Paragon. Subsequently, the increased performance of the sorbent demonstration indicates future designs will require less mass and volume than the current EDU.

The lunar testing proves out the feasibility of pure vacuum swing operation, making MTSA a technology that can be tested and used on the Moon prior to going to Mars.

Desorption of $\mathrm{CO}_{2}$ is very time dependent; a review of previously collected data displays a similar dependence that is elucidated by comparing the much larger cycle times seen during current testing to the shorter cycle times of previous work. The much longer time cycles of the current EDU as compared to the previous Paragon test articles (12 minute cycles vs. 50 second cycles for the Martian case) and the performance in the lunar testing suggest that we will achieve or exceed the sorbent performance levels that are required for Martian operation. It should be noted though, that testing is required to evaluate this expectation. The gathered thermocouple data, and the associated wave of warming seen in the bed, as well as the high sorbent loading indicates efficient bed loading, and closely mimics the model predictions. 


\section{Future Work}

As detailed herein, the work performed helped to greatly develop the MTSAS technology. Additionally it elucidated approaches for future work to mature this technology:

a. Calibration of the developed Thermal Desktop models ${ }^{6}$ to the tested Sorbent Bed design. The current design is based on sorbent models developed for use with earlier, lower performance tests. Sorbent model calibration based on current results will allow exploration of the identified mass and volume savings in the Mars capable unit.

b. While cyclic operations for set metabolic rates was tested, demonstration of cyclic operations that closely mimic an 8 hour EVA with varying metabolic rates was not tested, but is required to fully demonstrate operations of the MTSAS in a simulated lunar environment.

c. With lunar feasibility demonstrated, the next step is to configure and test the EDU in Martian conditions to demonstrate the feasibility of closed cycle temperature swing operation. Where practical the hardware necessary for this testing was included to allow the EDU to be readily tested under Martian conditions. This would allow better characterization of the response of beds of different length, and assessment of how the wash coating process has improved.

d. As demonstrated in Table 2 , the existing Mathcad system model (summarized in Ref 7) can be rapidly exercised to show the general impact of sorbent loading as well as other parameters that would be derived from Martian testing on MTSA design. Inputs from a correlated and validated Thermal Desktop model will allow further optimization by removing margins and unknowns in the system model. This may result in overall shrinkage of the MTSAS.

e. Currently the maximum $\mathrm{CO}_{2}$ concentrations leaving the sorbent bed are required to be below 33,000 ppm (7.6 $\mathrm{mm}-\mathrm{Hg}$ ) but current PLSS requirements are now using time averaged maximum of 16,500 ppm $(3.8 \mathrm{~mm}-\mathrm{Hg})$. Future efforts will need to take this into account when determining the required cycle time to begin the desorption half-cycle.

\section{Acknowledgments}

Paragon gratefully acknowledges funding for this work from NASA Johnson Space Center under Contract NNX09CB09C. The authors also want to acknowledge contributions from Walter Harrington, ensuring the timely and successful completion of testing.

\section{References}

${ }^{1}$ Iacomini, C., MacCallum, T., Morin, T., Straub-Lopez, K., and Paul, H. L., "Martian Liquid CO2 and Metabolic Heat Regenerated Temperature Swing Adsorption for Portable Life Support Systems", Space Technology and Applications International Forum (STAIF) 2007, Albuquerque, NM, February $11-15,2007$.

${ }^{2}$ Iacomini, C. S., Powers, A., Bower, C., Straub-Lopez, K., Anderson, G., MacCallum, T., and Paul, H. L., "Metabolic heat regenerated Temperature Swing Adsorption for CO2 \& Heat Removal/Rejection in a Martian PLSS", 37th International Conference on Environmental Systems, 2007-01-3273, 2007.

${ }^{3}$ Iacomini, C. S., Powers, A., Dunham, J., Straub-Lopez, K., Anderson, G., MacCallum, T., and Paul, H. L., "Demonstration of Metabolic Heat Regenerated Temperature Swing Adsorption Technology", 37th International Conference on Environmental Systems, 2007-01-3274, 2007.

${ }^{4}$ Iacomini, C. S., "Technology Development Program for Metabolic heat-regenerated Temperature Swing Adsorption (MTSA) for O2 Removal / Rejection in a Martian Portable Life Support System", Space 2011 Conference, AIAA -2011-7257.

${ }^{5}$ Iacomini, C. S., Powers, A., and Paul, H. L., "PLSS Scale Demonstration of MTSA Temperature Swing Adsorption Bed Concept for CO2 Removal/Rejection,” 2009-01-2388, 39th International Conference on Environmental Systems, Savannah, GA, July 12 -July 16, 2009.

${ }^{6}$ Bower, C. E., Padilla, S. A., Iacomini, C. S., and Paul, H., "Sorbent, Sublimation, and Icing Modeling Methods: Experimental Validation and Application to an Integrated MTSA Subassembly Thermal Model", 41st International Conference on Environmental Systems (ICES), Portland, OR, July 2011.

${ }^{7}$ Padilla, S. P., Iacomini, C. S., Powers A., Bower C. E., and Paul, H., "Design and Assembly of an Integrated Metabolic heat regenerated Temperature Swing Adsorption Subassembly Engineering Development Unit", $42^{\text {nd }}$ International Conference on Environmental Systems, San Diego, CA (submitted for publication).

${ }^{8}$ Padilla, S. P., Powers A., Ball, T., Iacomini, C. S., and Paul, H., "Investigation of Condensing Ice Heat Exchangers for MTSA Technology Development” 2009-01-2387, 39th International Conference on Environmental Systems, Savannah, GA, July 12 -July 16, 2009.

${ }^{9}$ Padilla, S. P., Iacomini, C. S., Powers A., and Paul, H., "Investigating Liquid Carbon Dioxide as a Coolant for an MTSA Heat Exchanger Design” 40th International Conference on Environmental Systems, Barcelona, Spain July 11 -July 15, 2010. 